\title{
O desempenho acadêmico como indicador de qualidade da transição Ensino Médio-Educação Superior
}

\author{
Caterine Vila Fagundes* \\ Maria Beatriz Luce** \\ Sebastián Rodriguez Espinar***
}

\section{Resumo}

Este artigo deriva de estudo e teve como objetivo propor um modelo de avaliação do desempenho acadêmico dos estudantes que transitam da Educação Básica para a Educação Superior a partir da abordagem teórica das transições. Mais especificamente, buscou-se conhecer fatores associados ao êxito na transição Ensino Médio-Educação Superior. Neste sentido, apresentam-se perspectivas de análise acerca do desempenho acadêmico, bem como diversas de suas definições ou conceitos. Os procedimentos metodológicos da pesquisa, por sua vez, são do tipo ex post fato e compreendem uma aplicação descritiva com enfoque preditivo e de desenvolvimento longitudinal. O modelodeavaliação proposto foi validadonos cursos de licenciatura emLetras, História, Matemática e Biologia da Universidade Federal do Rio Grande do Sul (UFRGS). Constatou-se, a partir de análise descritiva e de regressão, que os antecedentes dos alunos de Matemática e Biologia apresentam diferenças significativas com relação ao dos alunos de Letras e História; e que a formação recebida na educação básica, principalmente no Ensino Médio, é fator fundamental no desempenho acadêmico durante os dois primeiros semestres de estudos universitários.

Palavras-chave: Desempenho acadêmico. Educação Superior. Transição acadêmica.

\section{Introdução}

A partir da última década do século passado, evidencia-se substancial aumento do número de alunos na Educação Superior no Brasil, especialmente na rede

\footnotetext{
Doutora em Educação, Universidade Federal do Rio Grande do Sul- UFRGS, Núcleo de Estudos de Política e Gestão da Educação, Faculdade de Educação, E-mail: caterine.fagundes@gmail.com

** Doutora em Educação, Universidade Federal do Rio Grande do Sul - UFRGS, Núcleo de Estudos de Política e Gestão da Educação, Faculdade de Educação, E-mail:lucemb@ufrgs.br

*** Doutor em Educação Universidad de Barcelona - UB, Depto. de Métodos de Investigación y Diagnóstico en Educación, E-mail:srodriguez@ub.edu
} 
privada. Esse incremento ocorreu sem planificação detalhada ou processos efetivos de regulação e avaliação, questão que colocou em tela de juízo a qualidade da Educação. Em paralelo, ocorreu também ampla expansão do acesso à Educação Básica. São mudanças importantes do ponto de vista social e promissoras para o desenvolvimento do País, mas que implicam recepcionar, nas universidades, jovens com perfil sociocultural e educacional distinto - e, portanto, oferecer-lhes conteúdos e condições de aprendizagem significativamente diferentes dos usuais.

Sendo assim, o estudo da transição Ensino Médio-Educação Superior, em análise que contempla o processo educativo e diversos aspectos que permeiam a qualidade da educação, interessa à capacitação da ação educativa e à busca da excelência nas instituições de ensino e no sistema educativo. Nesse sentido, ressalta-se a importância, para as instituições de Educação Superior, da avaliação do desempenho acadêmico dos estudantes que ascendem a esse nível educacional.

Com base no exposto, neste texto procura-se dar relevo a um trabalho de pesquisa antes realizado ${ }^{1}$ (FAGUNDES, 2008), visando a ampliar o âmbito de investigação assim como o diálogo com a comunidade acadêmica interessada em Administração e Pedagogia Universitária.

Destaca-se, pois, que o objetivo do referido estudo foi o de propor um modelo de avaliação do desempenho escolar com indicadores de êxito acadêmico, tendo por base a teoria das transições e centrado no seguinte questionamento: Que fatores estão associados ao desempenho acadêmico dos alunos que transitam para a Educação Superior?

Para alcançar tal intento, se faz necessário apresentar a priori a teoria das transições (ainda pouco conhecida no meio acadêmico), analisar o desempenho como indicador de qualidade da transição, assim como descrever o modelo proposto de avaliação do desempenho. Após a introdução e discussão do aparato teórico, encontra-se a metodologia utilizada na análise dos dados assim como os resultados obtidos no estudo.

1 Tese de doutorado intitulada Transición Enseñanza Media-Universidad en el Sistema Educativo brasileño, realizada no Departamento de Métodos de Pesquisa e Diagnóstico em Educação da Universidade de Barcelona, sob a orientação do Prof. Dr. Sebastián Rodriguez Espinar, (2008). 


\section{Transição Acadêmica - 0 conceito de transição no âmbito da educação}

A abordagem das transições no âmbito da educação escolar requer, primeiramente, uma definição do conceito de transição, ainda que diversos autores o tratem segundo seus próprios critérios de análise e interpretação. Gimeno Sacristán (1997) resume transição como o momento e a experiência de viver a descontinuidade dentro da natural multiplicidade, da diversidade e da polivalência do mundo atual. Enquanto Bronfenbrenner (1987) a concebe a partir de uma perspectiva ecológica e enfatiza a origem da transição quando a posição do sujeito, no ambiente ecológico, é alterada como resultado de uma mudança de papel, de ambiente ou de ambos, e de forma simultânea. Para o grupo de pesquisa TRALS ${ }^{2}$ :

As transições incorporam três conceitos importantes: o conceito de mudança: toda a transição implica sair de um contexto e entrar em outro; o conceito de processo: durante a história do indivíduo, este está em constante transição; e o conceito de trajetória: a transição não é um processo irreversível e sim um processo que permite diferentes saídas ou vias aos que transitam (2002, p. 2).

Com relação à definição elaborada por TRALS (2002), apoiada em Fischer e Cooper (1990), cabe mencionar que uma transição agrega mudanças, enquanto mudanças nem sempre incluem uma transição, uma vez que esta implica processos e tem duração prolongada.

Segundo Gimeno Sacristán (1997), a trajetória em uma transição pode ser sincrônica - a que ocorre em um determinado tempo vital - ou diacrônica - a experiência e o momento de passar, sem poder voltar, de um estágio a outro, de um status a outro diferente, de um nicho que superamos ou que perdemos a outro diferente.

Desta forma e a partir da breve discussão teórica sobre o conceito, entendese a transição acadêmica como um processo de transformação, que implica descontinuidade na trajetória vital, aspecto que requer ou condiciona uma mudança de conduta, de papéis e/ou de ambiente, e que é mediada por fatores institucionais e sociais.

2 Grupo de Pesquisa da Universidade de Barcelona (Departamento de Métodos de Investigação e Diagnóstico em Educação) que investiga transições acadêmicas e mercado de trabalho. 


\section{A Transição do Ensino Médio para a Educação Superior}

No Brasil, o Ensino Médio tem como finalidade consolidar e aprofundar, em um período mínimo de três anos, os conhecimentos adquiridos no Ensino Fundamental e habilitar o estudante a prosseguir os estudos ou prepará-lo para a formação e o exercício profissional de nível técnico. Nesse período, etapa anterior e obrigatória à Educação Superior, deve-se adotar metodologias de ensino e de avaliação que estimulem a iniciativa dos estudantes (BRASIL, 1996, 2010, 2012a, 2012b).

Sobre a transição do Ensino Médio para a Educação Superior, Coromina e Isus explicitam que "o ingresso na universidade é a transição mais relevante da trajetória acadêmica dos alunos que alcançam este nível de formação" (2002, p. 164).

Cabe acrescentar que o processo de transição à Educação Superior tem um caráter de transformação multifatorial devido à intervenção de diferentes fatores sociais, culturais, individuais e acadêmicos. Figuera e Torrado (2000) definem essa transição como "um processo complexo que acarreta para o estudante múltiplas e significativas mudanças pessoais e vitais. Entre estas mudanças está a adaptação a um contexto educativo, regulado por normas explícitas e/ou implícitas que se deve conhecer para funcionar adequadamente" (2000, p. 2).

Nessa transição, coexistem fatores pessoais e ambientais que afetam o impacto da mudança no período específico em que ela ocorre, sendo esse impacto mediado pela natureza do apoio disponível e por estratégias de coping, ${ }^{3}$ com relação às mudanças, inerentes ao próprio indivíduo (SCHLOSSBERG, 1984). As características individuais dos estudantes assumem um papel mediador dos efeitos produzidos pelo contexto acadêmico no acesso e na adaptação à Educação Superior (PASCARELLA, TERENZINI, 1991). Por exemplo, diversos autores estão de acordo com a influência da universidade na aprendizagem e no desenvolvimento dos alunos, porém, ainda que seja positiva, esta não é determinante, sendo mediada por outras variáveis psicossociais dos estudantes, como seus projetos e interesses, suas estratégias ao enfrentar determinadas situações, seu status social ou seu gênero (ASTIN, 1997; CHAPMAN; PASCARELLA, 1983). Corominas e Isus (1998) salientam que

3 Em Psicologia, coping é o processo de gerenciamento que demanda esforços para solucionar problemas pessoais e interpessoais, buscando minimizar, reduzir ou tolerar esses conflitos (ENDLER; PARKER, 1990). 
universidades desenvolvem permanentemente programas de orientação acadêmica que incluem intervenções para facilitar o processo de inserção na universidade e, posteriormente, no mercado de trabalho. No entanto, no Brasil, ainda são incipientes as práticas nesse sentido, uma vez que a noção sobre a responsabilidade institucional pela articulação entre o Ensino Médio e a Educação Superior é recente.

Entretanto, no cenário latino-americano, já se encontram iniciativas no que se refere ao desenvolvimento de estudos sobre a transição Ensino Médio-Educação Superior, ainda que as articulações entre as investigações e a discussão sobre os critérios de análise adequados ao contexto sejam escassas ou incipientes. Cabe acrescentar que o desenvolvimento desses estudos tende a estar a cargo das instâncias responsáveis pelas estatísticas oficiais, que mostram diferentes aspectos dessa transição em relação a qualificações, distribuição dos egressos do Ensino Médio e desempenho escolar.

Contudo, atualmente existe um crescente interesse por parte dos governos, das instâncias internacionais e das agências de qualidade e de credenciamento em analisar e qualificar a transição do Ensino Médio para a Educação Superior. Pode-se afirmar que o estudo dessa transição está assumindo um importante significado no que se refere à qualidade no processo educativo. O desafio está posto aos professores universitários e ao conjunto dos profissionais que atuam nas instituições e na gestão das políticas públicas de Educação Superior; em decorrência, aos pesquisadores da área.

\section{0 desempenho acadêmico na transição Ensino Médio-Educação Superior}

A problemática do baixo desempenho acadêmico do estudante é um tema atual de debate, devido às repercussões que acarreta ao sistema educacional e ao mundo do trabalho. Segundo Rodríguez, Fita e Torrado (2004), no âmbito nacional, supõe-se um incremento crescente no gasto público com a Educação Superior. Já no âmbito universitário, pode resultar na redução do desempenho geral da instituição universitária, e em um número excessivo de alunos nas salas de aula, com estudantes que não concluirão o curso ou que empregarão mais tempo do que o necessário para obter a titulação. No âmbito individual, o baixo desempenho acadêmico tem como consequência o abandono ou o atraso nos estudos, o que é uma fonte provável de insatisfação pessoal, que gera deterioração da personalidade e frustração (LATIESA, 1986; POZO, HERNÁNDEZ, 1997). 
Considerando o impacto da Educação Superior nos estudantes, o desempenho acadêmico constitui também um potente indicador de qualidade de uma instituição. Assim, afirma a Commission on Higher Education: "O desempenho acadêmico dos estudantes é um indicador-chave para determinar se uma instituição está alcançando os seus objetivos" (1995, p. 5). Segundo argumenta Rodríguez (2003), "foi a perspectiva da qualidade como eficácia-eficiência econômica que fez com que a atenção aos logros se constituísse em um referencial fundamental na moderna avaliação da qualidade das universidades" (2003, p. 94). Daí a pertinência de se conceituar desempenho acadêmico e examinar suas diferentes perspectivas de análise.

\section{Notas teóricas e operativas acerca do desempenho acadêmico}

Estabelecer o conceito e o campo de aplicação do desempenho acadêmico não é tarefa simples. Tradicionalmente, foram elaboradas diferentes definições a respeito desse tema. Touron (1984) afirma que, em termos educativos, o desempenho é um dos resultados da aprendizagem, suscitado pela atividade educativa do professor e produzido no aluno, ainda que esteja claro que nem toda aprendizagem é produto da ação docente. Para Jiménez (2000), o desempenho acadêmico é concebido como um construto que não só contempla atitudes e motivação dos alunos, mas também outras variáveis intervenientes, como aspectos docentes, relação professor-aluno, entorno familiar, etc. Conforme Magalhães e Andrade (2006, p. 2), o desempenho acadêmico, está relacionado a fatores como inteligência, habilidade e competência. Não obstante, assegura Braga (2004, p.1) que "a avaliação do desenvolvimento e do aprendizado dos alunos, isto é, a determinação de quão bem os alunos alcançam os objetivos acadêmicos, é uma das principais maneiras pelas quais as instituições demonstram suas efetividades.".

Desta forma, essas definições amplas nos remetem à necessidade de buscar termos operacionais que possibilitem a compreensão e a análise do desempenho acadêmico. Gimeno Sacristán (1976), por exemplo, em seu estudo sobre a relação entre desempenho escolar e personalidade do aluno, estima que as notas, com certas reservas, são um instrumento valioso de comparação entre ambos os aspectos. Por sua vez, Latiesa (1992) diferencia o desempenho em sentido amplo (êxito, atraso e abandono) e em sentido restrito (notas). Vários 
autores concordam que, no desempenho em sentido restrito, as notas são o indicador mais viável para definir o desempenho acadêmico, "principalmente se as notas refletem os logros nos diferentes componentes (ou dimensões) do produto universitário (aspectos acadêmicos, profissionais e pessoais), ou seja, o perfil completo da formação" (RODRÍGUEZ, FITA, TORRADO, 2004, p. 3).

A partir desses pressupostos, pode-se entender a tendência de adotar o conceito de desempenho acadêmico como a relação entre o potencial (input) do estudante e o produto que se logra (aprendizagem).

A seguir analisam-se alguns estudos que contemplaram os resultados do produto ao final do processo de transição para a Educação Superior com o objetivo de selecionar as variáveis do modelo proposto.

\section{Perspectivas de análise acerca da transição do Ensino Médio-Educação Superior e o desempenho acadêmico}

Verifica-se que os estudos mais importantes sobre fatores associados ao desempenho acadêmico foram realizados durante a década de 1990, com maior incidência no decorrer dos anos 2000.

A partir da revisão dos estudos que compreendem a transição Ensino MédioEducação Superior, classificaram-se as investigações que analisam os elementos da transição a partir da natureza dos fatores de êxito. Tais estudos são agrupados segundo os modelos psicológico, sociológico, psicossocial e eclético de interação.

O modelo psicológico centra-se fundamentalmente nos fatores de habilidades e personalidade, que englobam as seguintes variáveis: coeficiente intelectual, habilidade numérica e raciocínio abstrato, pensamento lógico e verbal, características de personalidade, motivações, aspirações e expectativas, estilos cognitivos de aprendizagem e grau de satisfação. Esse modelo foi pioneiro nos estudos da transição para a Educação Superior e do desempenho acadêmico, sendo inúmeras as investigações que abordam as características psicológicas dos alunos e professores. Como exemplo, cita-se o estudo de Lahmers e Zulauf (2000) que revelou que as habilidades de gestão do tempo e o tempo dedicado aos estudos estavam positivamente associados com os resultados do Grade 
Average Point (GPA) ${ }^{4}$. Por sua vez, Somers, Cofer e Vander Putten (2002) afirmam que os pais tem um papel importante na escolha profissional dos filhos. Já o trabalho de Boulter (2002) estuda a relação entre autoconceito e sucesso acadêmico durante o primeiro ano do college. Tuckman (2003) combina o estudo das variáveis de motivação (autoconfiança, responsabilidade e gestão da vida) e as variáveis de aprendizagem (leitura, escrita, interpretação de textos). Em contexto brasileiro, Cunha e Carrilho (2005) concluem que o desempenho acadêmico pode ser afetado pelas vivências dos estudantes em nível pessoal e de realização acadêmica experimentadas no primeiro ano do curso superior. Araújo, Camargos e Carmagos (2011) encontraram que o desempenho acadêmico é afetado pelo número de faltas dos discentes, idade e sexo.

O modelo sociológico apresenta a dificuldade de isolar as variáveis determinantes devido à própria dimensão do contexto sociológico ou à dificuldade de medir e analisar tais fatores. A importância das variáveis ambientais sempre foi reconhecida, ainda quando não analisada, pois estas fazem referência aos modelos estruturais e estáticos dos contextos familiar, institucional e social e a seus aspectos dinâmicos. Essa linha investigativa assume que os estudantes trazem consigo uma variedade de atributos (sexo e capacidade), origem familiar (status social, expectativas familiares) e experiências pré-universitárias (sucesso no Ensino Médio e/ou em outros estudos prévios). Todos esses fatores, juntos ou separados, influem no desempenho acadêmico do estudante. Contudo, ainda não foi encontrado estudo, cujo modelo compreendesse exclusivamente a perspectiva de análise sociológica.

O modelo psicossocial baseia-se na crescente importância dos processos interpessoais mais próximos e imediatos ao próprio indivíduo. De acordo com Clemente, Albiñana e Doménech (1997), foram adotadas duas posturas generalizadas frente a esse modelo. Uma tentou examinar os processos desde um ponto de vista externo, observando como determinadas variáveis de estrutura (campo familiar ou escolar) ou de processo (atitudes dos demais para com o sujeito) atuavam sobre o indivíduo. Outra, tenta perceber essa mesma influência, mas, a partir de um marco de referência interno, através da percepção dos sujeitos sobre o significado e repercussão das referidas variáveis de estrutura e de processo.

4 O GPA significa a média geral de notas. Há dois tipos de GPA: Term GPA, a média de notas das disciplinas cursadas durante um semestre; o outro chamado de Overall GPA, que significa a média de notas de todas as disciplinas durante todo o curso. Neste modelo apresentado foi calculado o Term GPA do período analisado. 
Pode-se encontrar, com frequência, investigações que adotam ambas as posturas, como o estudo de Asbury (1974), no qual, entre os fatores que influem nas diferenças de desempenho, destaca: aspectos socioeconômicos, baixo nível de aspirações dos pais com relação aos filhos e baixo conceito de si mesmo por parte do sujeito como resultado de relações familiares deficientes. A obra de Benjamin e Hollings (1997), sobre a satisfação do estudante, conclui que a satisfação acadêmica está diretamente relacionada com a autoimagem positiva. Já os autores Pritchard e Wilson (2003) centraram-se nas seguintes variáveis para predizer o desempenho acadêmico: variáveis demográficas (sexo, idade, antecedentes educativos dos pais, média das notas do ensino médio, pontuações do exame de acesso à educação Superior e a classificação) e variáveis emocionais (estresse, perfeccionismo, autoestima, otimismo, fadiga, humor e depressão).

Woosley (2003), em sua investigação sobre fatores associados ao desempenho acadêmico, conclui que as variáveis ajuste social, suporte familiar, compromisso e constância nos estudos e a nota média do Ensino Médio têm um peso importante no sucesso da transição para a Educação Superior.

Em referência aos modelos e estudos anteriormente apresentados, Forner e outros autores (2000, p. 3 ) observam que:

As investigações se ocuparam em buscar as explicações do sucesso ou fracasso da transição nos próprios estudantes, sem considerar a influência dos sistemas de procedência (Ensino Médio) e de destino (Universidade), como se a responsabilidade da transição fosse do sujeito que transita e não dos sistemas pelos quais transita.

Os autores acrescentam ainda que, atualmente, no estudo das transições, aumenta-se o interesse por três dimensões muito concretas do fenômeno. A primeira é a dimensão pessoal dos grupos que transitam, as características dos estudantes, seus perfis pessoais, os itinerários curriculares, o aproveitamento dos estudos prévios, a distribuição do tempo, a gestão econômica, as expectativas, o apoio familiar, a confiança pessoal, etc. A segunda dimensão é a institucional e a do sistema educativo, ou seja, as condições e as características (oferta curricular, dependência administrativa, qualidade do ensino, etc.) das instituições, entre as quais transitam os estudantes, os sistemas que regulam tais instituições e o acesso. A terceira 
dimensão se refere à análise contextual em que se produz a transição: a influência e a relação com os ciclos socioeconômicos e sociodemográficos, com as políticas educativas e com as titulações de maior demanda no mercado de trabalho.

Com base em uma análise valorativa dos modelos anteriores concretizase o modelo eclético de interação. Esse modelo reconhece a influência das dimensões estáticas e dinâmicas da personalidade (inteligência, caráter, atitudes, motivações), e reconhece o valor determinativo de certas variáveis sociais, tanto de estrutura como de processo. Reconhece, principalmente, o valor do "eu" como elemento integrador e determinante da conduta. O modelo eclético de interação permite concluir que a transição Ensino Médio-Educação Superior, desde a perspectiva do desempenho acadêmico, da satisfação dos estudantes, com relação às suas conquistas aos processos de progresso e à adaptação ao meio, constitui um enfoque teórico mais adequado para explicar essa transição.

O estudo desenvolvido pelo grupo de pesquisa TRALS (2002) adotou tal modelo analisando a transição desde a perspectiva do desempenho acadêmico de duas coortes 1998-1999 e 1999-2000. Com a base de dados longitudinal, que abarcou os dois primeiros anos de estudos na Universidade de Barcelona, procuraram responder à pergunta: Qual o desempenho dos alunos que ingressaram na Universidade de Barcelona nos anos de 19981999 e 1999-2000? E, em que medida aparecem diferenças no desempenho de acordo com determinadas variáveis explicativas da persistência acadêmica? Os resultados revelam que o sexo um fator diferenciador no que se refere ao desempenho acadêmico, que os alunos que ingressam no curso desejado obtêm melhores resultados e que o desempenho prévio (nota de acesso) é um claro preditor do desempenho acadêmico nos dois primeiros anos de estudos universitários.

Sendo assim, a partir da revisão de alguns estudos anteriormente apresentados, pudemos constatar que os fatores de aptidão (habilidades) e de personalidade são os que aparecem frequentemente nos estudos como variáveis explicativas do sucesso na transição para a Educação Superior. Neste sentido, faz-se pertinente detalhar os fatores mais significativos para o êxito na Educação Superior. 


\section{Principais fatores explicativos do desempenho acadêmico:}

Destacam-se, a seguir, os fatores preponderantes como explicativos do desempenho acadêmico na transição Educação Básica-Educação Superior: motivação, autoconceito, metas acadêmicas, escolha do curso e notas de acesso.

\section{Motivação}

Nos estudos consultados, a análise da motivação está embasada nas atribuições dos alunos referentes aos seus próprios resultados e metas acadêmicas. Weiner (1986) formulou uma teoria da motivação na qual as atribuições do sujeito sobre seus próprios sucessos e fracassos desempenham um papel central. Nesta perspectiva, conforme Valle, González e Martinez (1999, p. 213), o que realmente determina a motivação são as distintas interpretações e valorações que o sujeito realiza dos seus próprios resultados acadêmicos". Por conseguinte, assume-se que a atribuição (ou explicação para os êxitos e fracassos acadêmicos próprios e alheios) tem grande representatividade no estudo da motivação.

\section{Autoconceito}

O autoconceito é reconhecido como o elemento mais importante no estudo do processo motivador (WEINER, 1990). Refere-se ao conjunto de percepções e crenças que uma pessoa tem sobre si mesma em diferentes áreas (SHAVELSON, HUBNER, STANTON, 1976), sendo um dos principais determinantes do desempenho dos alunos.

Entre as pesquisas sobre autoconceito as mais relevantes, como mencionado na investigação de Gonzalez-Pienda et al. (1997), são as de Marsh (1990), que afirma que o autoconceito determina casualmente o desempenho dos alunos; as de Chapman, Lambourne e Silva (1990); e as de Skaalvik e Hagtvet (1990), que asseguram ser as experiências de êxito acadêmico que determinam o autoconceito dos alunos.

\section{Metas acadêmicas}

Quanto aos objetivos acadêmicos dos alunos, as pesquisas (DWECK, 1986; DWECK: LEGGETT, 1988; NICHOLLS, 1984; AMES, 1992) procuram explicar a motivação com base nas metas do sujeito, as quais são determinadas, em parte, pela noção que o sujeito tem de sua capacidade. 
A maioria dos autores concorda que uma das metas mais importantes é a competência percebida (GONZALEZ, VALLE, PÉREZ, 1996; GONZÁLEZPIENDA et al., 1997). Nesse estudo, os autores fazem uma revisão do conceito de meta acadêmica e sua relação com a motivação escolar. Os objetivos perseguidos pelos alunos, que determinam o modo como enfrentam suas atividades acadêmicas, podem ser agrupados em quatro categorias:

- metas relacionadas com as tarefas,

- metas relacionadas com a autopercepção,

- metas relacionadas com a valoração social,

- metas relacionadas à obtenção de recompensas externas.

Cada tipo de meta tem uma orientação motivacional de caráter intrínseco ou extrínseco. Mas são dois tipos de objetivos, de aprendizagem (intrínsecos) e desempenho (extrínsecos), que se subdividem em metas de reforço social e metas de desempenho.

Os diferentes padrões de motivação, que existem em relação ao desempenho, são determinados pelo tipo das metas de realização escolhidas pelo sujeito.

\section{Escolha do curso}

Com relação à dimensão escolha do curso superior, o ajuste entre a carreira desejada e a cursada é um bom preditor da persistência na Educação Superior (Blinne, Wayne e Johnston, 1998). Rodriguez, Fita e Torrado (2004) concluem que uma adequada escolha do curso é indicador de permanência na Universidade:

Os fatores que mais influenciam na persistência acadêmica são os correspondentes ao desempenho prévio ao acesso à Universidade. Neste caso, analisam o promeio das notas do Ensino Médio, a nota obtida no exame final de Ensino Médio e a nota de acesso à Universidade, que foram fornecidas pelos próprios alunos (p. 12).

Apodaka e Gallarreta (1999), por sua vez, apresentam a taxa bruta de alunos vocacionalmente satisfeitos (escolha do curso e curso em que estão matriculados) como um indicador associado à taxa de desempenho, e afirmam que o sistema de acesso baseado em numerus clausus pode distorcer a conhecida associação 
do desempenho a fatores pessoais e sociais como motivações, interesses, expectativas de sucesso profissional, etc.

\section{Nota de acesso}

Diversos autores - como Escudero (1987), Latiesa (1992), e Rodríguez, Fita e Torrado (2004) - consideram que esta é a variável de maior peso nos resultados acadêmicos, ao menos nos dois primeiros anos de estudos na universidade. Independentemente do debate acerca dos componentes e do modo de obtenção desta nota, a mesma constitui um indicador básico do desempenho prévio à Educação Superior e, em todos os casos, de transições acadêmicas. De acordo com Rodriguez, Fita e Torrado (2004), o desempenho da etapa anterior é o melhor preditor do desempenho inicial na Universidade.

Por fim, conforme assevera Herrera (1999, apud RODRÍGUEZ, FITA, TORRADO, 2004), o melhor desempenho acadêmico se evidencia nos alunos com alto proveito no Ensino Médio, com uma alta valoração nos seus hábitos de estudo, que assistem regularmente às aulas, com alto grau de satisfação com o curso escolhido, motivados culturalmente pelo contexto familiar e com um conceito elevado de autoeficácia.

\section{Objetivos e procedimentos metodológicos}

Do ponto de vista da abordagem do problema, a pesquisa realizada é quantitativa, do tipo ex-post-facto concretizando-se em um estudo descritivo, através da aplicação de questionários, com um enfoque preditivo. Segundo Gil (2009), a tradução literal da expressão ex-post-facto é: "a partir do fato passado". Portanto, significa que, nesse tipo de pesquisa, o estudo foi realizado após a ocorrência de alterações na variável dependente. Nesse sentido, tem-se uma investigação sistemática, na qual o pesquisador não controla diretamente as variáveis independentes porque já ocorreram suas manifestações ou porque são intrinsecamente não manipuláveis. $\mathrm{Na}$ coleta de dados, uma peculiaridade citada por Moreira (2002) para esse tipo de pesquisa consiste no fato de, inicialmente, ser realizado um levantamento amostral, visando a coletar informações que serão utilizadas para descrever, comparar ou explicar fatos. Com isso, todo histórico de acontecimentos inerentes às variáveis são verificados a priori. Posteriormente há a coleta de dados, conforme a definição da pesquisa (GOMES et al. 2011). Neste caso, segundo esclarece Moreira (2002), como não é possível manipular as variáveis independentes conforme a necessidade 
da investigação, surge a necessidade de se encontrar em grupos que apresentem características semelhantes.

À vista disso, desenvolveu-se o estudo descritivo por questionário, com enfoque preditivo (ou correlacional) e de desenvolvimento longitudinal, dada a sequência temporal do processo de transição para a Educação Superior: início dos estudos, término do primeiro semestre e conclusão do primeiro ano de estudos. A pesquisa descritiva, segundo Gil (1988, p. 39), visava a descrever as características da determinada população ou do fenômeno como relações entre as variáveis.

Para conhecer as variáveis do desempenho acadêmico foi realizada uma análise preditiva que se classifica, segundo Bartolomé (1978), na associação entre variáveis. O tipo de regressão utilizado foi a múltipla stepwise. De acordo a Abbad e Torres (2002, p. 23):

A regressão stepwise geralmente é a estratégia escolhida para estudos exploratórios. Quando se está utilizando este tipo de regressão, o pesquisador, desprovido de uma teoria consistente sobre os fenômenos estudados, está interessado apenas em descrever relacionamentos pouco conhecidos entre variáveis, e não em os explicar. Neste tipo de regressão, a seleção da seqüência de entrada dos preditores na equação é feita estatisticamente, sem um modelo teórico consistente a ser seguido. Em estudos exploratórios, o pesquisador elabora um modelo teórico de investigação que inclui hipóteses sobre relacionamentos entre variáveis, mas que ainda não possibilita afirmações consistentes sobre a magnitude ou direção desses relacionamentos.

Cabe acrescentar que ao modelo stepwise regression foi aplicado um enfoque de regressão-predição diferencial (amostra de Ciências e Humanidades), dados os resultados descritivos deste estudo.

\section{Os sujeitos}

O modelo apresentado nesse estudo foi validado com alunos que ingressaram na Universidade Federal do Rio Grande do Sul (UFRGS) nos cursos de licenciatura em Matemática, Letras, História e Biologia. Foram questionados 227 alunos do total da amostra composta por 312 sujeitos, que completaram as vagas disponíveis no primeiro semestre letivo, aprovados no concurso vestibular. Os alunos que 
participaram do estudo foram os que estavam presentes em sala de aula no momento da aplicação do primeiro questionário. Não houve perda da amostra no decorrer do estudo. Para realizar as análises, foram agrupados os quatro cursos em duas áreas do conhecimento: Humanidades (representada pelos cursos de História e Letras)

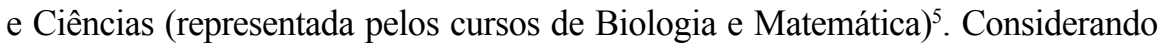
o total da amostra (população) e o total de alunos questionados, a margem de erro dos resultados está estimada em 3,5\%, conforme pode ser observado na Tabela 1:

\section{Tabela 1- Alunos que participaram do estudo}

\begin{tabular}{l|l|l|l|l}
\hline Curso & População & Amostra & \% de respostas & \% erro \\
\hline Biologia & 50 & 41 & 82 & 7 \\
\hline Matemática & 45 & 33 & 73 & 9 \\
\hline Total Ciências & 95 & 74 & 78 & 5,5 \\
\hline Letras & 132 & 86 & 65 & 6 \\
\hline Historia & 85 & 67 & 79 & 6 \\
\hline Total Humanidades & 217 & 153 & 70 & 4 \\
\hline Total Ciências + Humanidades & 312 & 227 & 73 & 3,5 \\
\hline
\end{tabular}

Fonte: Os autores (2014).

\section{Os instrumentos}

A primeira parte deste estudo foi do tipo exploratório de abordagem quantitativa, uma vez que o intuito foi produzir conhecimento que permitisse uma predição, explicação e entendimento dos fatores associados ao desempenho acadêmico (ou êxito acadêmico) dos alunos nos dois primeiros semestres de estudos na Educação Superior. Para a realização do trabalho utilizaram-se três questionários construídos pelo grupo de pesquisa TRALS $(2000)^{6}$. O primeiro instrumento foi submetido a um processo de validação em contexto brasileiro e posteriormente utilizado nessa pesquisa. Os outros

5 Cabe explicitar que o desigual número de vagas ofertadas nos diferentes cursos nos conduziu à sua agrupação em duas áreas do conhecimento, com o propósito de lograrmos uma amostra mais representativa para as análises dos dados.

6 A validação do conteúdo, os indicadores de consistência interna e as análises fatoriais dos questionários elaborados pelo grupo TRALS podem ser consultados em: TRALS. Informe transición secundaria-universidad, Universidad de Barcelona, 2000. Alguns dos dados mais significativos são: o total de variáveis estão agrupadas em 138 questões, a maioria do tipo fechada, com escala de valoração do tipo Likert (valoração entre 1 e 5 pontos). Utilizou-se análise fatorial-técnica analítica multivariada-, com o objetivo de reduzir as 138 perguntas em menos fatores ou dimensões. 
dois questionários apenas sofreram adaptações linguísticas. O primeiro instrumento tratou de coletar os dados referentes ao acesso à universidade estabelecendo dimensões em relação às características sociodemográficas, socioculturais e econômicas, a qualidade na escolha do curso e a experiência educativa prévia ao acesso à universidade. $\mathrm{O}$ segundo questionário recolheu dados relativos ao processo de estudo durante o primeiro semestre na universidade, fundamentalmente com relação à avaliação dos alunos a respeito de seu próprio desempenho. O terceiro questionário, por sua vez, teve o propósito de coletar dados referentes à inserção dos alunos na universidade após um ano de estudos.

Com Hernández, Collado e Baptista (1991), que definem o questionário como um conjunto de perguntas sobre uma ou mais variáveis a serem medidas (categorias a descrever), entendeu-se que este é um instrumento com capacidade de coletar informações variadas em uma única aplicação. No caso, o questionário foi um recurso útil por sua aceitação conjunta de seções estruturadas, semiestruturadas e abertas (CABRERA; ESPIN, 1986).

\section{Procedimentos}

Neste estudo longitudinal, consideraram-se três momentos fundamentais da coleta de informações: ao início dos estudos na universidade, ao final do primeiro semestre e ao final do segundo semestre de estudos. A aplicação do primeiro questionário ocorreu presencialmente, com prévia autorização da Pró-Reitoria de Graduação da UFRGS, dos coordenadores dos cursos e dos professores, enquanto os alunos estavam em sala de aula. Embora a coleta tenha sido realizada em contexto coletivo, solicitou-se que os alunos respondessem ao questionário individualmente. Cabe ressaltar que a aplicação do segundo e terceiro questionários ocorreu via Internet, diretamente pelo e-mail de cada estudante. Periodicamente e de modo coletivo, através de um mailing list, contatava-se com os alunos para solicitar que, voluntariamente, expusessem questões referentes ao processo de estudos. Essas questões não foram incluídas na análise da investigação, mas foram utilizadas como forma de manter contato com os alunos e a amostra íntegra.

\section{Análise dos dados}

As técnicas de análise dos dados, utilizadas para responder à pergunta de partida, ou seja, que fatores estão associados ao sucesso na transição Ensino 
Médio-Universidade?, foram a descritiva e diferencial (de estatística descritiva: porcentagem, média e desviação típica) para caracterizar a amostra e descrever o processo de transição durante os dois semestres de estudos e a análise de regressão. Para realizar tais análises foi usado o programa de análise estatística SPSS e o modelo stepwise de regressão. O objetivo desta etapa de trabalho (análise de regressão) foi obter uma equação de predição apoiada na relação entre duas variáveis (preditora e dependente) ou em diversas variáveis (preditoras e dependentes). Sendo assim, o modelo linear hipotetizado a partir das considerações teóricas foi: $Y^{\prime}=B 1 X 1+B 2 X 2+e$, onde $Y^{\prime}$ representa a variável dependente; $X 1$, uma das variáveis intependentes, $X 2$, outra variável independente (e assim sucessivamente de acordo às variáveis cujo aporte demonstre-se significativo). O coeficiente B pondera o aporte de cada uma das variáveis do modelo; o valor "e" é o valor do erro máximo de estimativa, que se refere à diferença entre os valores observados e preditos. Para que o uso desta equação seja eficaz na predição da variável dependente em estudo, observouse previamente os pressupostos da regressão múltipla. Sendo assim, para cada equação de regressão consideraram-se:

a) Os valores do coeficiente de correlação múltipla $(\mathrm{R})^{7}$, do coeficiente de determinação $\left(\mathrm{R}^{2}\right)$ e do coefíciente de determinação ajustado $\left(\left(\mathrm{R}_{\mathrm{a}}{ }_{\mathrm{a}}\right)\right.$, em virtude do número de preditores e do tamanho da amostra);

b) os valores do erro máximo de estimativa (e);

c) os valores de significância estatística $(\mathrm{F})$;

d) a porcentagem total da variância do critério que se explica através dos preditores incluídos na equação;

e) as variáveis ou preditores inicialmente incluídas no modelo;

f) os valores dos coeficientes B e Beta para cada uma das variáveis com aporte significativo;

Por outro lado, as informações obtidas através dos questionários permitiram algumas conclusões sem a necessidade de utilizar técnicas de contraste ou estatística inferencial.

Estimou-se que os fatores que configuram esse modelo permitiriam melhor conhecimento do processo de acesso à Educação Superior a partir do perfil dos

7 Para comprovar se existe relação entre um grupo de variáveis independentes com a variável dependente, recorremos ao coeficiente de correlação múltipla $(R)$, cujos valores variam entre $(0 \mathrm{a}+1)$. 
alunos que acessam a esse nível educativo, dos antecedentes que facilitam a transição do Ensino Médio para a Educação Superior, da percepção dos alunos com relação ao novo ambiente educativo, dos indicadores de adaptação nesse contexto, bem como das variáveis que estão associadas ao êxito no acesso à universidade.

\section{Resultados e discussão}

Apresentam-se, a seguir, as principais características ou especificidades da amostra selecionada, os resultados depois de um ano de estudos na Educação Superior, bem como os fatores que condicionam o desempenho acadêmico dos estudantes na Universidade.

\section{Perfil de acesso dos alunos: a preparação para afrontar os estudos}

Descrevemos a amostra a partir de três dimensões: características sociodemográfica; antecedentes socioculturais e econômicos; antecedentes escolares.

Cabe acrescentar, no entanto, que os dados foram apresentados, primeiramente, com base numa análise global das áreas do conhecimento e, comparativamente, quando ocorreram diferenças significativas entre as áreas de Ciências e Humanidades.

\section{1) Características sociodemográficas}

As características como sexo, idade e tipologia da instituição de origem nos brindam com informações básicas sobre os alunos. Essas informações podem explicar as diferenças na transição do Ensino Médio para a Educação Superior. Em relação ao sexo dos alunos que ingressam na UFRGS, ambas as áreas do conhecimento estão igualmente representadas por estudantes do sexo masculino e feminino. A principal diferença sócio-demográfica dos alunos que acedem aos cursos de licenciatura da UFRGS tem a ver com a idade. Um total de $55 \%$ dos estudantes tem mais de 20 anos, e não se observam diferenças estatísticas por área do conhecimento. Este dado é coerente com a problemática da distorção idade/série no Ensino Médio, tema constantemente discutido e analisado no Brasil devido ao ingresso tardio no sistema escolar, a repetição de séries e o abandono dos estudos por temporadas. Essa situação, analisada por Avanzini (1994), pode ser um fenômeno condicionante do baixo desempenho acadêmico. 
Quanto à tipologia das instituições de ensino de procedência dos alunos, observou-se que $51 \%$ dos alunos de ambas as áreas do conhecimento provêm de instituições privadas. Não foram observadas diferenças significativas segundo a área do conhecimento.

\section{2) Antecedentes socioculturais e econômicos}

Os antecedentes familiares e econômicos ajudam a explicar a transição dos alunos para a universidade. Por essa razão é necessário considerar alguns pontos relacionados, tais como os estudos do pai e da mãe, o apoio familiar, a manutenção e financiamento dos estudos. Pitchard e Wilson (2003) constataram que existe uma série de variáveis preditoras do desempenho académico, entre elas os antecedentes educativos dos pais e familiares.

No contexto educativo desta investigação, a maioria dos alunos entrevistados (72\%) formam parte da segunda geração na Educação Superior; 58\% dos pais dos alunos de Ciências têm educação superior; enquanto que os pais dos alunos de Humanidades perfazem 47\%; Dos alunos entrevistados, 78\% dependem economicamente da família ( $84 \%$ dos alunos de Ciências e $62 \%$ dos alunos de Humanidades). A análise de médias para amostras independentes mostrou que os pais dos alunos de Ciências têm maior interesse cultural do que os pais dos alunos de Humanidades, e que, com relação ao apoio familiar, os primeiros, demonstram maior preocupação com os estudos dos filhos.

\section{3) Antecedentes escolares}

Os alunos foram questionados sobre suas percepções acerca da experiência educativa prévia ao acesso à Educação Superior. Assim verificamos quais os antecedentes que facilitaram o acesso dos mesmos que ingressaram na UFRGS, de acordo com alguns critérios descritos a seguir:

- Qualidade da escolha do curso

Segundo Rodríguez (2003), a dimensão "qualidade da escolha do curso" pode ser analisada a partir da trajetória educativa dos estudantes ou das informações e objetivos sobre o curso escolhido; e afirma, ainda, que a escolha adequada dos estudos é um indicador de permanência na Universidade. Dessa forma, foram considerados aspectos relacionados aos estudos anteriores, aos objetivos com o curso escolhido e as influências nesta escolha. 
A maioria dos alunos entrevistados, (ou seja, 66\%), afirma ter ingressado pela primeira vez na Educação Superior. Sobre os objetivos dos alunos com o curso escolhido, a partir de uma escala de 1 a 5 (pouco e muito), a variável aquisição de conhecimento para exercer a profissão escolhida foi avaliada como fundamental $(4,84)$, e não foram observadas diferenças significativas entre as áreas do conhecimento. Com relação às influências na escolha do curso, o fator conexão com os estudos e as características pessoais foi os mais estimados pelos alunos $(4,41)$, sem diferenças significativas entre o grupo. A respeito da satisfação com o curso escolhido $90,5 \%$ dos alunos mostraram-se satisfeitos.

- Experiência educativa prévia à Educação Superior

Com o intuito de verificar a experiência educativa prévia da amostra participante do estudo, os alunos responderam a um conjunto de questões sobre a valoração de alguns aspectos referentes à formação no Ensino Médio, medido através da escala Likert de 1 a 5 (insuficiente e excelente). Os alunos valoraram o item desenvolvimento de atitudes e valores como "bom" $(3,51)$, com um desvio padrão de $(1,122)$. Os itens: satisfação com a escola, capacitação nas diferentes disciplinas, capacidade de análise crítica, capacitação nas técnicas de estudo e realização dos trabalhos de casa, o clima de trabalho e a motivação em sala de aula foram avaliados como "regular" (3). Finalmente, a aprendizagem de línguas foi o item avaliado como "ruim" $(2,45)$ com um desvio padrão relativamente elevado $(1,181)$.

Na comparação de médias para amostras independentes entre as duas áreas do conhecimento, observou-se que os estudantes de Ciências avaliam mais positivamente a experiência na instituição de origem. Cabe acrescentar que Woosley (2003) constatou que a adequada preparação para aceder à Educação Superior tem correlação positiva com o desempenho acadêmico. Nesse estudo, a maioria dos alunos pesquisados, isto é $69 \%$, considerou importante realizar aulas de reforço, além da escola, para ingressar na Universidade. Averiguou-se que $81 \%$ dos alunos de Ciências tiveram aulas de reforço dos conteúdos aprendidos na escola para aprovação no exame de acesso à Universidade, enquanto que $63 \%$ dos estudantes de Humanidades tiveram aulas de reforço. A formação recebida no Ensino Médio se constitui em uma base, tanto teórica como procedimental e atitudinal, que permite aos 
alunos lidar com a sua integração na Educação Superior. Neste estudo, 52\% dos alunos consideram-se satisfeitos com a formação recebida na escola. Dos alunos provenientes da escola pública, apenas $31 \%$ estão satisfeitos com a sua formação, enquanto que, $73 \%$ dos estudantes da rede privada, estão satisfeitos com a formação educacional recebida.

- Nota de acesso à Universidade

Analisou-se a nota de acesso dos estudantes de acordo com a área do conhecimento e a tipologia da instituição de origem. Ao realizar a comparação entre as médias de acesso, de acordo com o tipo de instituição a partir da qual os alunos se originaram, observou-se que existem diferenças significativas. Alunos oriundos das instituições privadas têm uma nota de acesso superior a dos alunos provenientes das instituições públicas, como se pode observar na Tabela 2:

Tabela 2 - Média da nota de acesso à Universidade por tipo de instituição de origem e área do conhecimento

\begin{tabular}{|c|c|c|}
\hline Pública e Humanidades & Privada e Humanidades & Subtotal \\
\hline$X=557,99$ & $X=572,77$ & $X=565,2746$ \\
\hline$S=46,64$ & $S=53,80$ & $S=50,64456$ \\
\hline$N=73$ & $N=71$ & $N=144$ \\
\hline Pública e Ciências & Privada e Ciências & Subtotal \\
\hline$X=556,98$ & $x=600,65$ & $X=580,7428$ \\
\hline$S=59,24$ & $S=49,44$ & $S=58,00993$ \\
\hline$N=31$ & $N=37$ & $N=68$ \\
\hline Subtotal & Subtotal & Total \\
\hline$X=557,6872$ & $X=582,3002$ & $X=570,2361$ \\
\hline$S=50,43103$ & $S=5378510$ & $S=53,48696$ \\
\hline$N=104$ & $N=108$ & $N=212$ \\
\hline
\end{tabular}

Fonte: Os autores (2014). 
- Motivação para afrontar os estudos

De acordo a teoria da motivação de Weiner (1986), o que realmente determina a motivação são as diferentes interpretações e avaliações que um indivíduo faz de seus próprios resultados acadêmicos. Uma grande porcentagem dos alunos, (ou seja, 72\%), manifesta-se muito motivada para começar os estudos universitários, assim como não foram observadas diferenças nas duas áreas do conhecimento.

A transição para a Educação Superior assume um importante significado no que diz respeito à qualidade do sistema educativo. Consideram-se os indicadores da transição para a Universidade como potentes indicadores da qualidade dos sistemas de Ensino Médio e da Educação Superior; e podemos relacionar as competências aprendidas na escola como elementos básicos do avanço, em termos de conhecimento, que pode ser alcançado durante os estudos na universidade. No presente estudo, evidenciou-se que os alunos de Ciências provêm de um ambiente sociocultural e econômico mais favorável que os das Humanidades, bem como apresentam nota de acesso superior. É lícito afirmar também que os alunos da rede privada se sentem mais satisfeitos com a formação recebida no Ensino Médio.

Trajetória acadêmica nos dois primeiros semestres de estudos universitários: os resultados depois de um ano de estudos na Educação Superior

Após o primeiro ano de estudos, é possível medir o êxito da transição, ou seja, a partir dessa fase podemos concluir tanto sobre a permanência dos alunos na universidade como o desempenho acadêmico dos mesmos. Corominas (2002) menciona que, ao final do primeiro ano de estudos na Educação Superior, se completa a transição do ensino médio à universidade, por ser, nesse período que se constituem as diferentes trajetórias da transição ${ }^{8}$.

- A permanência na Universidade após o primeiro ano de estudos

Segundo a pesquisa realizada, verificou-se que a permanência dos estudantes após os dois primeiros semestres de estudos é alta: $82 \%$ dos estudantes continuam matriculados na mesma universidade e no mesmo curso; $5 \%$ dos

8 Diferentes trajetórias: continua no mesmo curso e na mesma instituição; mudou de instituição, mas segue no mesmo curso; trocou de curso, mas segue na mesma instituição; trocou de curso e de instituição; abandonou os estudos. 
alunos continuaram na universidade, porém em outro curso; $2,5 \%$ permaneceram no mesmo curso, mas em outra instituição, e 3,5\% dos alunos abandonaram os estudos. De igual forma, não foram observadas diferenças significativas entre as duas áreas do conhecimento.

- O Desempenho dos alunos no primeiro ano de estudos na Universidade

Com relação ao desempenho acadêmico após dois semestres de estudos observa-se que o $\mathrm{GPA}^{9}$ dos alunos das Humanidades é superior ao dos alunos de Ciências, como se pode observar na Tabela a seguir:

Tabela 3 - GPA referente aos dois primeiros semestres de estudos na universidade

\begin{tabular}{l|l|l|l|l}
\hline Área de conhecimento & média & desvio padrão & erro padrão & amostra \\
\hline Ciências & 3,84 & 0,6841 & $(0,0949)$ & 74 \\
\hline Humanas & 4,18 & 0,6571 & $(0,0647)$ & 153 \\
\hline Total & & & & 227 \\
\hline
\end{tabular}

Fonte: Os autores (2014).

\section{Um modelo de predição do desempenho ao final do segundo semestre de estudos universitários}

De acordo com o momento inicial dos estudos na universidade, e por meio de aspectos relacionados à evolução/transcurso dos dois primeiros semestres de estudos do estudante, complementa-se a análise com os resultados finais do desempenho ao término do segundo semestre de estudos. Para responder à pergunta que norteia esta pesquisa, foi proposto um modelo de predição no qual estão representadas as variáveis de análise da transição, dentre as quais se destacam os inputs de acesso, os fatores mediadores e os produtos da transição marcados por diferentes momentos (antes, durante e depois).

9 O GPA significa a média geral de notas. Há dois tipos de GPA: Term GPA, a média de notas das disciplinas cursadas durante um semestre; o outro chamado de Overall GPA, significa a média de notas de todas as disciplinas durante todo o curso. Neste modelo apresentado foi calculado o term GPA do período analisado (2 semestres). Calculou-se o GPA dos alunos a partir do número de créditos de cada disciplina multiplicado pela nota obtida (nota entre 1 e 5 pontos, de acordo à qualificação adotada pela Universidade: $A ; B ; C ; D ; E$ ). Posteriormente dividiu-se a pontuação obtida pelo número de créditos matriculados. 
- Modelo proposto

O modelo de avaliação do desempenho acadêmico, concebe a transição como um processo que inicia no Ensino Médio (preparação), edifica-se no primeiro semestre de estudos na Educação Superior e culmina com o "que se logra" (aprendizagem). Centra-se, portanto, no primeiro ano de estudos na universidade.

As variáveis escolhidas para o modelo foram as que, com base na revisão da literatura anteriormente exposta, apareceram em diversos estudos como preditoras do desempenho acadêmico. Sendo assim, as variáveis incluídas no modelo foram:

- Variável dependente:

- Desempenho acadêmico

- GPA

- Variáveis independentes (ou preditoras):

a) Suporte social

- Antecedentes socioculturais e econômicos

- Apoio familiar

b) Competências acadêmicas prévias

- Qualidade da escolha do curso

- Experiência educativa prévia

- Nota de acesso

c) Motivação e expectativas dos alunos

- Autoconceito

- Metas acadêmicas

Para responder ao questionamento proposto foi realizada a análise de regressão múltipla stepwise com a amostra de Matemática e Biologia (Ciências) e a de Letras e História (Humanidades).

Observa-se no Quadro 1 a síntese dos dados significativos para a interpretação dos resultados da pesquisa: 


\section{Quadro 1 - Síntese dos dados significativos para a interpretação dos resultados}

\begin{tabular}{|c|c|c|c|c|}
\hline $\begin{array}{l}\text { VARIÁVEL } \\
\text { DEPENDENTE } \\
\text { (GPA) }\end{array}$ & VALORES & VARIÁVEIS INDEPENDENTES & B & Beta \\
\hline CIÊNCIAS & $\begin{array}{l}R=0,607 \\
R 2=0,369 \\
\text { R2a ajustado =0,353 } \\
\text { erro máx. estimativa } \\
(e)=0,46734 \\
\text { significância }(F)= \\
0,000 \\
\% \text { variância critério } \\
=35,3\end{array}$ & $\begin{array}{l}\text { 1. antecedentes sociocultu- } \\
\text { rais e econômicos } \\
\text { 2. apoio familiar } \\
\text { 3. Escolha do curso } \\
\text { 4. experiência educativa } \\
\text { prévia } \\
\text { 5. nota de acesso } \\
\text { 6. autoconceito } \\
\text { 7. metas acadêmicas }\end{array}$ & 0,006 & 0,607 \\
\hline $\begin{array}{l}\text { HUMANIDA- } \\
\text { DES }\end{array}$ & $\begin{array}{l}R=0,474 \\
R 2=0,224 \\
\text { R2a ajustado =0,205 } \\
\text { erro máx. estimativa } \\
(e)=0,53533 \\
\text { significância }(F)= \\
0,003 \\
\% \text { variância critério } \\
=20,5\end{array}$ & $\begin{array}{l}\text { 1. antecedentes sociocultu- } \\
\text { rais e econômicos } \\
\text { 2. apoio familiar } \\
\text { 3. qualidade da escolha do } \\
\text { curso } \\
\text { 4. experiência educativa } \\
\text { prévia } \\
\text { 5. nota de acesso } \\
\text { 6. autoconceito } \\
\text { 7. metas acadêmicas }\end{array}$ & $\begin{array}{l}0,035 \\
0,004\end{array}$ & $\begin{array}{l}0,339 \\
0,302\end{array}$ \\
\hline
\end{tabular}

Fonte: Os autores (2014).

Para a amostra de Ciências, ao introduzir as variáveis previstas, o modelo confirma somente uma variável como preditora do desempenho acadêmico: a nota de acesso $(0,607)$. Cabe esclarecer que o valor de $\mathrm{R}(0,607)$ é moderado, portanto estatisticamente significativo. $\mathrm{O}$ valor da $\mathrm{R}^{2}$ ajustada é $(0,353)$, o que indica que a nota de acesso explica $35,3 \%$ da variância da variável GPA. A equação que permite estimar a pontuação do GPA é a seguinte:

GPAciências $=0,006$ nota de acesso $+0,46734$

Considerando a amostra de Humanidades, ao introduzir as variáveis previstas, o modelo confirma duas variáveis como preditoras do desempenho acadêmico (os valores de Beta indicam a ordem da intensidade da aportação): a nota de acesso $(0,302)$ e a experiência educativa prévia $(0,339)$. Nesse caso, o valor de R $(0,474)$ é moderado, e estatisticamente significativo. Em consequência, é moderada a explicação da variância da variável dependente (20,5\%). A nota de acesso e a experiência educativa prévia explicam $20,5 \%$ de variação do GPA na 
amostra de Humanidades. A equação de predição de GPA a partir das variáveis preditoras para a amostra de Humanidades é:

GPAhumanidades $=0,035$ experiência educativa prévia $+0,004$ nota de acesso $+0,53533$

Evidencia-se o desigual comportamento das diferentes variáveis na amostra de Humanidades e Ciências (20,5\% vs. 35,3\% de explicação da variância). A nota de acesso, em que pese a sua desigual ponderação, é um preditor significativo nos dois modelos. A experiência educativa prévia aos estudos na universidade, indicador de natureza cognitiva, aparece em segundo lugar. Tais aspectos manifestam a confirmação, uma vez mais, do histórico peso dos preditores: habilidades e desempenho prévio (nota do exame de acesso) na predição do desempenho nos primeiros semestres de estudos na educação superior com relação à variável critério GPA.

Os resultados deste estudo evidenciam, portanto, que o sistema de procedência (Ensino Médio) e um ambiente cultural favorável são determinantes no que diz respeito ao êxito acadêmico. Além de sugerir medidas de orientação, cujos modelos de intervenção englobem a realidade atual do Ensino Superior, que incluam o indivíduo e a instituição, e que trate, entre outros fatores, da diversidade dos alunos em relação à idade e à origem dos diferentes contextos educativos, tendo em conta os novos papéis e funções a serem desempenhados pela Educação Superior.

\section{Considerações finais}

Constatou-se que os antecedentes dos alunos de Matemática e Biologia apresentam diferenças elevadas e significativas com relação aos alunos de Letras e História, no que diz respeito ao maior nível de estudos dos pais, mais apoio familiar e mais assistência aos cursos preparatórios para o vestibular. Porém, essas diferenças constatadas não influenciaram no desempenho dos alunos, pois observou-se uma nota média (GPA) mais alta na amostra de Letras e História. Contudo, falta um dado para concluir que as exigências, no processo avaliativo das disciplinas dos cursos de Matemática e Biologia, sejam maiores do que nos cursos de Letras e História. Conclusões mais consistentes, nesse teor, dependem de análise dos critérios de avaliação de cada curso e de cada disciplina, fora do alcance desse estudo. 
Considerando os indicadores da transição para a universidade como potentes evidências da qualidade do Ensino Médio e da Educação Superior, assume-se que a formação recebida na Educação Básica, fundamentalmente no Ensino Médio, são elementos fundamentais no progresso que os estudantes podem alcançar durante os dois primeiros semestres de estudos na universidade.

Parece razoável pensar que, se foi determinado estatisticamente, no contexto do modelo, que várias variáveis explicam uma parte da variância da variável dependente, se soubermos com antecedência as pontuações dos sujeitos nessas variáveis independentes, poder-se-á antecipar a pontuação do GPA dos alunos. Porém, é evidente, e de acordo com os estudos anteriormente analisados, que faltam elementos para que se conheça com precisão o desempenho dos alunos.

$\mathrm{O}$ acesso aos estudos superiores constitui um processo complexo no qual interagem múltiplos fatores e agentes educativos. Há evidências - e, ousa-se dizer, consenso geral - de que a pesquisa sobre a Educação Superior no Brasil é ainda incipiente, seja em termos das dinâmicas do sistema nacional ou das políticas públicas que tem sido experimentadas, como da gestão das instituições e suas ações visando a qualidade e à produtividade acadêmica. Nesse sentido, cumpre advertir sobre a falta de tratamento das informações disponíveis, como de pesquisas institucionais que avancem sobre áreas que ainda não são objeto dos sistemas estatísticos, ou não são próprias para tal.

Da mesma sorte, ocorro no incipiente esforço de formação acadêmica para tais empreendimentos, pois tem sido raras as teses e dissertações que trabalham sobre as bases de dados disponíveis ou se aventuram na coleta e tratamento de informações em escala larga ou institucional. Evidencia-se, pois, a falta de mais experiências e referências que permitam comparar e avaliar os estudos, que possibilitem um conhecimento rigoroso a par da complexidade do sistema educativo e social atual.

Esta problemática geral dificulta a ação orientadora e educativa nesse campo, e a elaboração de propostas coerentes e efetivas para a transição, bem como limita as próprias conclusões do estudo que se toma como core deste artigo.

As transições acadêmicas não se devem examinar e enfrentar somente desde a perspectiva estritamente acadêmica (de desempenho), mas também 
incorporando outras variáveis que incidem no processo. É preciso criar um ambiente de colaboração entre todos os agentes implicados, que garanta uma ação orientadora de qualidade. Ressalte-se que os resultados e a efetividade da aplicação do modelo de avaliação do desempenho acadêmico proposto podem contribuir para intervenções de qualificação da Educação Superior, seja no âmbito das políticas públicas ou da gestão institucional.

Por outro lado, para melhor compreensão do fenômeno, reconhece-se a importância de um modelo multimetodológico que integre a abordagem qualitativa na avaliação do desempenho acadêmico, e inclua fatores que caracterizem os objetivos e o desenvolvimento da educação no contexto estudado, com o intuito de lograr um maior conhecimento da complexa, plural e multifacetária realidade humana e, desta forma, produzir descrições, quantificações, explicações ou interpretações críticas, válidas, mais precisas e confiáveis. Ainda, pensa-se ser fundamental incluir na análise outros fatores que participam desse processo, como, por exemplo, o corpo docente, e avaliar suas implicações na referida transição. Ademais, evoluir para estudos comparativos entre os subsistemas federativos e entre redes escolares ou universitárias no Brasil, como entre países com os quais estão sendo estabelecidas redes de cooperação e mobilidade acadêmica.

\section{Referências}

ABBAD, G.; TORRES, C. V. Regressão múltipla stepwise e hierárquica em Psicologia Organizacional: aplicações, problemas e soluções. Estudos de Psicologia,[S. 1.], n. 7, p. 19-29. 2002.

ARAÚJO, E. A. T.; CAMARGOS, M. A.; CAMARGOS, M. C. S. Desempenho acadêmico dos discentes do curso de ciências contábeis: uma análise dos seus fatores determinantes em uma IES privada. In: ENCONTRO DA ANPAD ENANPAD, 35., 2011, Rio de Janeiro. Anais ... Rio de Janeiro: Associação Nacional de Pós-Graduação e Pesquisa em Administração, 2011.

AVANZINI, G. El fracaso escolar. Barcelona: Herder, 1994.

AMES, C. Achievement goals, motivational climate, and motivational processes. In: ROBERTS, G. C. (Ed.). Motivation in sport and exercise. Champaign: Human Kinetics Publishers, 1992. p.161-176. 
APODAKA, P.; GALLARRETA, L. Propuesta de dos indicadores del acceso/demanda de estudios universitarios. In: VIDAL, J. (Coord.). Indicadores en la Universidad: información y decisiones. Madrid: Consejo de Universidades; MEC, 1999.

ASBURY, C. A. Selected factors influencing over and under achievement in young school-age children. Review of Educational Research, [S.1.], v. 34, p. 44- 66, 1974.

ASTIN, A. How good is your institution retention rate. Research in Higher Education, [S.1.], v. 38, p. 647-657, 1997.

BARTOLOMÉ, M. Estudios correlacionales y predictivos. Barcelona: Univar, 1978.

BENJAMIN, M.; HOLLINGS, A. Student satisfaction: test of an ecological model. Journal of College Student Development, [S.1.], v. 38, n. 3, p. 213- 228, 1997.

BOULTER, L. T. Self-concept as a predictor of college freshman academic adjustment. College. Student Journal, [S.1.], v. 36, n. 2, p. 234-246, 2002.

BRAGA, D. B.; FRANCO, L. R. H. R. Revolucionando as técnicas de aprendizagem da engenharia com o EAD. In: World Congress on Engineering and Technology Education, São Paulo: [s.n.], p.1083-1087, 2004.

BRASIL. Lei 9.394, de 20 de dezembro de 1996. Lei de Diretrizes e Bases da Educação Nacional . Diário Oficial da União, Brasília, DF, 23 dez. 1996. Seção 1, p. 2.7833.

. Ministério da Educação. Conselho Nacional de Educação. Parecer homologado sobre processo n. 23001.000196/2005-41. Diretrizes Curriculares Nacionais Gerais para a Educação Básica. Diário Oficial da União, 9 set. 2010, Brasília, DF, Seção 1, p. 10.

. Ministério da Educação. Conselho Nacional de Educação. Parecer homologado sobre processo n. 23001.000189/2009-72. Diretrizes Curriculares Nacionais para o Ensino Médio. Diário Oficial da União, 24 dez. 2012a, Brasília, DF, Seção 1, p. 10. 
BRASIL. Ministério da Educação. Conselho Nacional de Educação. Resolução n. 2, de 30 de janeiro de 2012. Define diretrizes Curriculares Nacionais para o Ensino Médio. Diário Oficial da União, Brasília, DF, 31 jan. 2012b, Seção 1, p. 20.

BRONFENBRENNER, U. La ecología del desarrollo humano. Barcelona: Paidós, 1987.

CABRERA, F.; ESPIN, J. Medición y evaluación educativas. Barcelona: PPU, 1986.

CHAPMAN, D. W.; PASCARELLA, E. T. Predictors of academic and social integration of college students. Research in Higher Education, [S.1.], n. 19, p. 295- 321, 1983.

CHAPMAN, J. W., LAMBOURNE, R.; SILVA, P. A. Some antecedents of academic self-concep: a longitudinal study. British Journal of Educational Psychology, [S.1.], n.60, p.142-152, 1990.

CLEMENTE, A.; ALBIÑANA, P.; DOMÉNECH, F. Predicción del Rendimiento Académico en estudiantes de secundaria a través de los factores de inteligencia, socialización, desadaptación escolar, autoconcepto y personalidad. Revista de Psicología de la Educación, Barcelona, n. 22, p. 55-68, 1997.

COMMISION ON HIGHER EDUCATION. Framework for outcomes assesment. Middel State Association, 1995.

COROMINAS, E. R.; ISUS, S. Transiciones y Orientación. Revista de Investigación Educativa. Barcelona, v.16, n. 2, p. 155-184, 1998.

COROMINAS, E. R. La transición a los estudios universitarios. Abandono o cambio en el primer año de universidad. Revista de Investigación Educativa. Barcelona, v. 19, n.1, p. 127-151, 2002.

CUNHA, S. M.; CARRILHO, D. M. O processo de adaptação ao ensino superior e o rendimento acadêmico. Psicologia Escolar e Educacional, [S.1.], v.9, n. 2, p. 215-224, 2005. 
DWECK, C. Motivational processes affecting learning. American Psychologist, [S.1.], v. 41, n.10, p.1040-1048, 1986.

DWECK, C. S.; LEGGETT, E. L. A social-cognitive approach to motivation and personality. Psychological Review, [S.1.], v. 95, p.256-273, 1988.

ENDLER, N. S.; PARKER, J. D. A. The multi-dimensional assessment of coping: a critical evaluation. Journal of Personality and Social Psychology, [S.1.], v. 42, p. 207-220, 1990.

ESCUDERO, T. Experiencias evaluativas en la Universidad de Zaragoza. In: Varios (Eds.). Consideraciones Metodológicas sobre Evaluación y Mejora de la Docencia Universitaria. Valencia: Servei de Formació Permanent; Universitat de València, 1987.

FAGUNDES, C. Transición enseñanza media-universidad en el sistema educativo brasileño. 2008. 231p. Tese (Doutorado)-Faculdade de Educação, Universidad de Barcelona, Barcelona, 2008.

FORNER, A. et al. La transición Secundaria-Universidad: los alumnos de LOGSE: Comunicación en el 1r Congreso Internacional Docencia Universitaria e Innovación. Barcelona: ICE's/UB/ UAB /UPC, 2000. CD-ROM.

FIGUERA, P.; TORRADO, M. El proceso de transición de bachillerato a la Universidad: Factores de éxito. Quaderns Institucionals, Barcelona, n. 2, p. 41$55,2000$.

FISCHER, S.; COOPER, C. L. On the Move: the psychology of change and transition. Chichester: John Wiley \& Sons, 1990.

GIL, A.C. Como elaborar projetos de pesquisa. São Paulo: Atlas, 1988. . Métodos e técnicas de pesquisa social. 4. ed. São Paulo: Atlas, 2009.

GIMENO SACRISTÁN, J. Autoconcepto, sociabilidad y rendimiento escolar. Madrid: MEC, 1976. 
GIMENO SACRISTÁN, J. La transición a la educación secundaria. Madrid: Morata, 1997.

GONZÁLEZ, R., VALLE, A., PÉREZ, J. C., et al. Una aproximación teórica al concepto de metas académicas y su relación con la motivación escolar. Psicothema, [S.1.], v.8, n. 1, p. 45- 61, 1996.

GONZÁLEZ-PIENDA, J. et al. Autoconcepto, autoestima y aprendizaje escolar. Psicothema, [S.1.], v. 9, n. 2, p. 271- 289, 1997.

HERNÁNDEZ; COLLADO; BAPTISTA. Metodología de la investigación. México: McGRAW-HILL, 1991.

JIMÉNEZ, M. Competencia social: intervención preventiva en la escuela. Infancia y sociedad. Universidad de Alicante, [S.1.], v. 24, p. 21-48, 2000.

LAHMERS, A. G.; ZULAUF, C. R. Factors associated with academic time use and academic performance of college students: a recursive approach. Journal of College Student Development , [S.1.], n. 41, p. 544-556, 2000.

LATIESA, M. Estudio longitudinal de una cohorte de alumnos de la universidad Autónoma de Madrid - análisis de la deserción universitaria. In: LATIESA, M. (Comp.). Demanda de Educación Superior y rendimiento académico en la Universidad. Madrid: CIDE-SGCU, p. 399-441, 1986 b.

. La deserción universitaria. Madrid: C.I.S., 1992.

MAGALHÃES, F. A. C.; ANDRADE, J. X. Exame Vestibular, características demográficas e desempenho na Universidade: em busca de fatores preditivos. In: CONGRESSO USP DE CONTROLADORIA E CONTABILIDADE, 6 , 2006. São Paulo. Anais... São Paulo: FEA/USP, 2006.

MARSH, H. W. Causal ordering of academic self-concept and academic achievement: a multiwave, longitudinal panel analysis. Journal of Educational Psychology, [S.1.], v. 82, p. 646-656, 1990.

MOREIRA, D. M. O método fenomenológico na pesquisa. São Paulo: Pioneira Thomson, 2002. 
NICHOLLS, J. G. Conceptions of ability and achievement motivation. In: AMES, R. ; AMES, C. (Ed.). Research on motivation in education. New York: Academic Press, 1984, p. 39-73.

PASCARELlA, E. T.; TERENZINI, P. T. How College Affects Students. San Francisco: Josey Bass, 1991.

POZO, C.; HERNÁNDEZ, J. M. El fracasso Académico en la Universidad: Propuesta de un modelo de explicación e intervención preventiva. In: APODACA; LOBATO (Ed.). Calidad en la Universidad: orientación y evaluación. Barcelona: Laertes, 1997, p. 137-152.

PRITCHARD, M. E.; WILSON, G. Using emotional and social factors to predict student success. Journal of College Student Development, [S.1.], v. 44, p. 18-27, 2003.

RODRÍGUEZ, S. La problemática del rendimiento en la transición secundariauniversidad. In: Actas X Jornadas sobre el acceso a la universidad. Sevilla: Universidad de Sevilla, p. 93- 113, 2003.

RODRÍGUEZ, S.; FITA, E.; TORRADO, M. El rendimiento académico en la transición secundaria- universidad. Revista de Educación, [S.1.], n. 334, p. 391414, 2004.

SCHLOSSBERG, N. Counseling adults in transition. New York: Springer Publishing Company, 1984.

SHAVELSON, R. J.; HUBNER, J. J.; STANTON, J. C. Self concept: validation of construct interpretations. Review of Educational Research, [S.1.], v. 46, n. 3, p.407- 441, 1976.

SKAALVIK, E. M.; HAGTVET, K.A. Academic achievement and selfconcept: an analysis of causal predominance in a developmental perspective. Journal of Personality and Social Psychology, [S.1.], n. 58, p. 292-307, 1990.

SOMERS, P., COFER, J.; VANDER PUTTEN, J. The early bird goes to college: The link between early aspirations and postsecondary attendance. Journal of College Student Development, [S.1.], v. 43, n. 1, p. 93-107, 2002. 
TOURON, J. Factores del rendimiento académico en la Universidad. Pamplona: EUNSA, 1984.

TRALS (Grup de Recerca sobre Transicions Acadèmiques i Laborals). Informe Transición Secundaria-Universidad. Barcelona: Universitat de Barcelona. Ejemplar fotocopiat, 2000.

. El rendiment acadèmic i la trajectória acadèmica en els dos primers anys de la universidad. Barcelona: Universitat de Barcelona, 2002.

TUCKMAN, B.W. The effect of learning and motivation strategies training on college students' achievement. Journal of College Student Development, [S.1.], v. 44, n. 3, p. 430-437, 2003.

VALLE, A., GONZÁLEZ, R., MARTINEZ, S., et al. Atribuciones causales, auto concepto y motivación en estudiantes con alto y bajo rendimiento académico. Revista Española de Pedagogía, Madrid, [S.1.], n. 214, p. 525-546, 1999.

WEINER, B. An attributional theory of achievement motivation and emotion. Psychological Review, [S.1.], v. 92, n. 4, p. 548-573, 1986.

. History of motivational research in education. Journal of Educational Psycology, [S.1.], v. 82, p. 616- 622, 1990.

WOOSLEY, S. A. How important are the first few weeks of college? The long term effects of initial college experiences. College Student Journal, [S.1.], v. 37, p. 201-207, 2003.

\section{The academic performance as a quality indicator from the High School transition to Higher Education \\ Abstract}

This study aims to propose a model to evaluate the academic performance of students who pass the Basic Education to Higher Education from the theoretical approach of the transitions. More specifically, we seek to know indicators associated with the success in the transition from the High School to the Higher 
Education. Thus, this article addresses the analytical perspectives on academic performance as well as their differing definitions or concepts. The methodological research procedures are the type ex post facto, and understand the application of the descriptive method with a predictive approach and longitudinal development. The proposed evaluation model was validated in bachelor degree of Languages, History, Mathematics and Biology at the Federal University of Rio Grande do Sul (UFRGS). In fact, what was found, from descriptive and regression analysis, was that the background of the students of Mathematics and Biology presents significant differences with regard to students of Languages and History, and the education received in the basic level, especially in High School is a fundamental factor in academic performance during the first two semesters of the university studies.

Keywords: Academic performance. Higher Education. Academic transition.

\section{El Rendimiento Académico como Indicador de Calidad de la Transición entre la Enseñanza Secundaria y la Educación Superior} Resumo

Este artículo deriva de estudio que tuvo por objetivo proponer un modelo de evaluación del rendimiento académico de los estudiantes que transitan de la Enseñanza Básica para la Educación Superior a partir del abordaje teórico de las transiciones. Especialmente, se ha buscado conocer los factores asociados al éxito en la transición entre la Enseñanza Secundaria y la Educación Superior. En este sentido, nuestro articulo se centra en las perspectivas de análisis sobre el rendimiento académico, así como en sus diversas definiciones o conceptos. Ya los procedimientos metodológicos de la investigación son del tipo ex post fato, y abarcan la aplicación del método descriptivo con un enfoque predictivo y cuyo desarrollo es longitudinal. El modelo de evaluación propuesto ha sido validado en los cursos de licenciatura de Letras, Historia, Matemáticas y Biología de la Universidad Federal de Rio Grande do Sul (UFRGS). Se ha constatado, a partir de análisis descriptivo y de regresión, que los antecedentes de los alumnos de Matemáticas y Biología presentan diferencias significativas con relación a los alumnos de Letras e Historia; y que la formación recibida en la Educación Básica, principalmente, en la Enseñanza Secundaria, es factor fundamental en el rendimiento académico durante los dos primeros semestres de estudios universitarios.

Palabras-clave: Rendimiento académico. Educación Superior. Transición académica. 